\title{
Perspectivas docentes ante necesidades de educación especial inclusiva en la UACAM: un estudio de caso
}

\author{
Teaching perspectives in the need of inclusive special education at Uacam. A \\ case study
}

\section{Perspectivas de ensino frente às necessidades de educação especial inclusiva na}

UACAM: um estudo de caso

\section{Martin Alberto Sosa Zumárraga}

Universidad Autónoma de Campeche, Facultad de Humanidades, México

marasosa@uacam.mx

https://orcid.org/0000-0002-2950-083X

\section{Resumen}

El objetivo de esta investigación fue determinar cuáles son las perspectivas de los docentes de la Universidad Autónoma de Campeche (UACAM) en torno a las necesidades de una educación especial inclusiva en dicha casa de estudios. Para ello, se empleó una metodología cualitativa, la cual permitió un primer acercamiento al problema de estudio. Para ello se diseñó una encuesta bajo el formato de la técnica de Likert que incluyó tres dimensiones (social, institucional y didáctica) que fueron construidas con base en 87 ítems que ofrecían cinco opciones de respuesta. Para este trabajo, sin embargo, solo se ofrecen los resultados de 14 ítems significativos que proporcionan información en torno a la perspectiva de los docentes hacia la compresión de la diversidad, así como las necesidades de cambios en la política educativa y de transformación en la práctica docente. En la investigación participaron 11 profesores (tres hombres y nueve mujeres) de la referida institución. De manera general, se puede concluir que si bien los profesores están a favor de iniciativas y cambios normativos que promueven la inclusión y la mejor atención posible a alumnos con NEE, también son conscientes de que trabajar con ellos puede ser difícil. De hecho, 


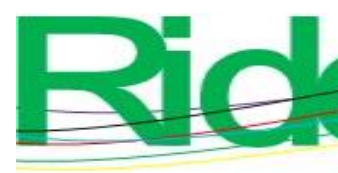

Revista Iberoamericana para la Investigación y el Desarrollo Educativo ISSN $2007-7467$

muchos señalan que hay confusión en el momento de atender a personas con estas características, por lo que se les dificulta la elaboración de un plan de trabajo.

Palabras claves: educación especial, equidad, estudios de género, inclusión educativa, Universidad de Campeche.

\section{Abstract}

The research objective was to determine the teacher's perspective of the Autonomous University of Campeche (UACAM) regarding of a special education needs I said this institution. For this study, a qualitative methodology was used, which allowed a first approach to the study problem. For this a survey was designed using the Likert technique format that included three dimensions (social, institutional and didactic) that were constructed based on 87 items that offered five response options.

For this investigation work, however, only the 14 significant items were taken, that offered information about the teacher's perspective towards to the understanding of diversity, as well as the needs for changes in educational policy and transformation in teacher's academic practice.

Eleven professors (three men and nine women) for the aforementioned institution participated in the research. In general, it can be concluded that although teachers are in favor of initiatives and regulatory changes that promote inclusion and the best possible care for students with special education needs (SEN), they are also aware that working with them can be difficult. In fact, many point out that there is confusion when caring for people with these characteristic, making it difficult for them to draw up a work plan.

Keywords: special education, equity, gender studies, inclusive education, University of Campeche.

\section{Resumo}

O objetivo desta pesquisa foi verificar quais as perspectivas dos professores da Universidade Autônoma do Campeche (UACAM) quanto às necessidades de uma educação especial inclusiva na referida casa de estudos. Para tanto, utilizou-se uma metodologia qualitativa, que permitiu uma primeira abordagem do problema de estudo. Para isso, foi elaborada uma pesquisa no formato da técnica Likert que contemplou três dimensões (social, institucional e didática) que foram construídas a partir de 87 itens que ofereciam cinco opções de resposta. Para este trabalho, entretanto, são oferecidos apenas os resultados de 14 itens significativos que fornecem informações sobre a perspectiva dos professores em relação à compreensão da diversidade, bem como as 


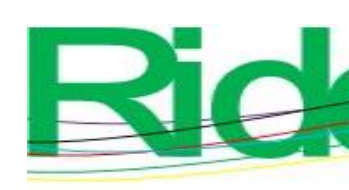

Revista Iberoamericana para la Investigación y el Desarrollo Educativo ISSN $2007-7467$

necessidades de mudanças na política educacional e de transformação na prática docente. Participaram da pesquisa onze professores (três homens e nove mulheres) da referida instituição. De forma geral, pode-se concluir que embora os professores sejam a favor de iniciativas e mudanças regulatórias que promovam a inclusão e o melhor atendimento possível aos alunos com NEE, também estão cientes de que trabalhar com eles pode ser difícil. De fato, muitos apontam que há confusão no atendimento a pessoas com essas características, dificultando a elaboração de um plano de trabalho.

Palavras-chave: educação especial, equidade, estudos de gênero, inclusão educacional, Universidade de Campeche.

Fecha Recepción: Diciembre 2020

Fecha Aceptación: Junio 2021

\section{Introducción}

La educación especial es una opción académica que surge con el propósito de sustituir a las escuelas tradicionalmente excluyentes y discriminadoras por espacios donde todos tengan las mismas oportunidades, sin importar el género, la edad, el origen social o étnico, la condición económica o las necesidades especiales de aprendizaje (Romero y García, 2013). En esa intención, por supuesto, la educación especial ha tenido que adaptarse a los marcos políticos, sociales, económicos e ideológicos de cada momento (De Landsheere, 1996; Toscano, Ponce, Cruz, Zapién, Contreras y Pérez, 2017; Vergara, 2002; García, 2018)) con el fin no solo de "garantizar a todos y todas el acceso libre y gratuito a los distintos niveles educativos, sino además de la posibilidad de transitarlos exitosamente" (Miguel e Indart, 2015, p. 458). Por ello, Vergara (2002) afirma que la historia de la educación especial es también la historia de la antropología cultural, pues se debe recordar que desde siempre han existido personas con necesidades especiales.

Ahora bien, aunque esa necesidad de una educación equitativa sea una premisa ampliamente divulgada y aceptada (Unesco, 2015, citada por Toscano et al., 2017), la realidad demuestra que aún falta mucho por hacer para consolidar ese ideal.

Por ejemplo, en el caso de la Universidad Autónoma de Campeche (UACAM) (institución donde se desarrolla la presente investigación), se puede indicar que dentro de su Plan Institucional de Desarrollo se señalan las intenciones de ofrecer una educación de calidad, flexible, inclusiva, con vinculación social e investigación aplicada, y con clara rendición de sus cuentas. Estas eran algunas de exigencias para mejorar significativamente la capacidad de responder a una sociedad del conocimiento y para vincularse con ejes estratégicos del Programa Sectorial de Educación que 


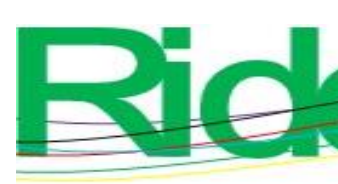

Revista Iberoamericana para la Investigación y el Desarrollo Educativo ISSN $2007-7467$

procuraban la erradicación de la pobreza y la asistencia a grupos vulnerables (UACAM, 20152019).

Sin embargo, en ninguno de los ejes o vertientes estratégicos del Plan Institucional de Desarrollo (UACAM, 2015-2019) ni en las estrategias de acción específicas se especifican las acciones que se deben desarrollar para atender a personas con NEE. Por este motivo, el propósito de esta investigación fue determinar cuáles eran las perspectivas de los docentes de la UACAM en torno a las necesidades de una educación especial inclusiva en dicha casa de estudios.

\section{La educación y las políticas educativas universitarias}

Al efectuar cambios o reformas en las políticas educativas deben quedar claros los objetivos trazados para percibir con claridad el logro de los aprendizajes proyectados, así como la garantía en las condiciones de acceso, permanencia, inclusión y participación de todos los alumnos, incluidos aquellos con necesidades educativas especiales [NEE] (Aguilar, 2019; p. 2). Esas reformas, por supuesto, también deben incluir modificaciones radicales en cuanto a las concepciones anacrónicas de profesores, autoridades educativas y demás factores relacionados con la educación en cuanto a que las diferencias individuales (físicas, psicológica, sociales, etc.) son un hecho inherente a la condición humana (Palomares, 1993). Por ejemplo, para la condición de retraso mental se debe saber que actualmente se define como una característica asociada a un rendimiento intelectual que se halla significativamente por debajo de la media (Ortiz, 1999).

En este sentido, y debido al aumento de alumnos con NEE en los niveles de educación media superior y educación superior, se debe ser consciente de que todos los seres humanos son distintos, de ahí que se deba procurar brindar una atención lo más personalizada posible (Aranda, 2002, p. 21).

Esa incidencia de estudiantes con NEE se refleja dentro de casi todas las unidades académicas de la UACAM, según la información ofrecida por su portal de transparencia, la cual fue solicitada para conocer la cantidad de alumnos con capacidades diferentes y especiales durante los periodos anuales de 2015 a 2019 (fases 1 y 2), así como la relación de cursos para la capacitación docente institucional dentro de este rubro (tabla 1 y figura 1 ). 
Tabla 1. Alumnos con capacidades diferentes y especiales en los periodos anuales de 2015 a 2019, en las fases 1 y 2

\begin{tabular}{|l|r|r|r|r|r|r|r|r|r|r|}
\hline \multicolumn{1}{|c|}{ Nivel Educativo } & $\begin{array}{r}\text { Fase } \\
15-1\end{array}$ & $\begin{array}{r}\text { Fase } \\
15-2\end{array}$ & $\begin{array}{r}\text { Fase } \\
16-1\end{array}$ & $\begin{array}{r}\text { Fase } \\
16-2\end{array}$ & $\begin{array}{r}\text { Fase } \\
17-1\end{array}$ & $\begin{array}{r}\text { Fase } \\
17-2\end{array}$ & $\begin{array}{r}\text { Fase } \\
18-1\end{array}$ & $\begin{array}{r}\text { Fase } \\
18-2\end{array}$ & $\begin{array}{r}\text { Fase } \\
19-1\end{array}$ & $\begin{array}{c}\text { Fase } \\
19-2\end{array}$ \\
\hline Bachilleratos & 209 & 188 & 330 & 320 & 299 & 296 & 430 & 362 & 458 & 413 \\
\hline $\begin{array}{l}\text { D.E.S. Ciencias } \\
\text { Agropecuarias }\end{array}$ & 0 & 0 & 0 & 0 & 0 & 0 & 10 & 8 & 18 & 14 \\
\hline $\begin{array}{l}\text { D.E.S. Ingenierías y } \\
\text { Ciencias }\end{array}$ & 3 & 3 & 4 & 4 & 10 & 9 & 109 & 96 & 215 & 200 \\
\hline $\begin{array}{l}\text { D.E.S. Ciencias } \\
\text { Sociales y } \\
\text { Humanidades }\end{array}$ & 4 & 3 & 0 & 3 & 9 & 3 & 195 & 175 & 381 & 342 \\
\hline D.E.S. Salud & 4 & 4 & 8 & 5 & 18 & 12 & 201 & 164 & 346 & 298 \\
\hline
\end{tabular}

Fuente: Plataforma Nacional de Transparencia. Infomex-Campeche: Folio 0100111920

Figura 1. Incidencia de alumnos con NEE

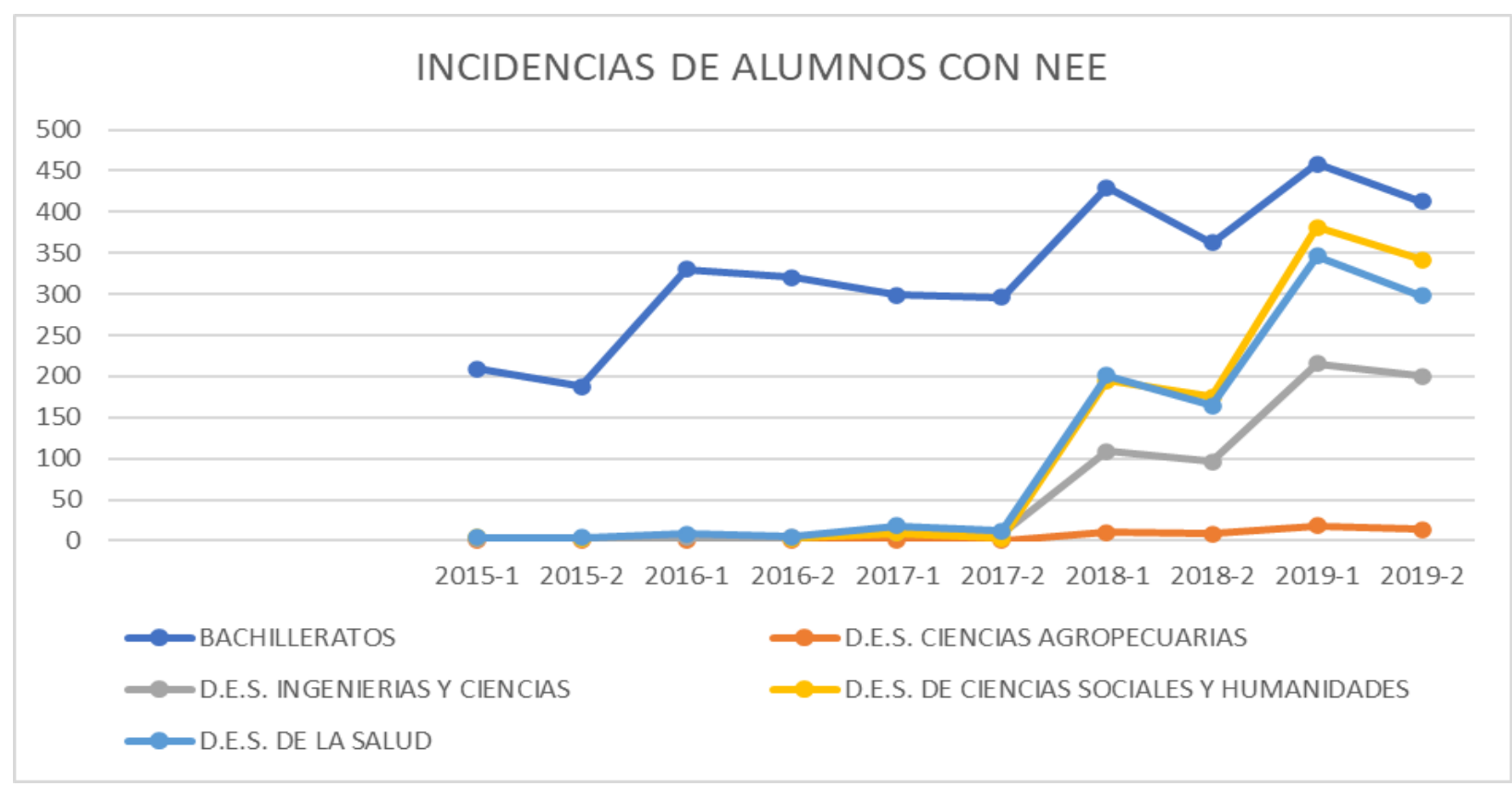

Fuente: Plataforma Nacional de Transparencia. Infomex-Campeche: Folio 0100111920

Sobre la información anterior, es preciso resaltar el incremento de alumnos con NEE en el plano educativo universitario y que no se aplican criterios en los registros específicos ante el área de control escolar durante su ingreso, permanencia o egreso, por lo que solo se expresan en términos generales basados en la discapacidad de las personas. 

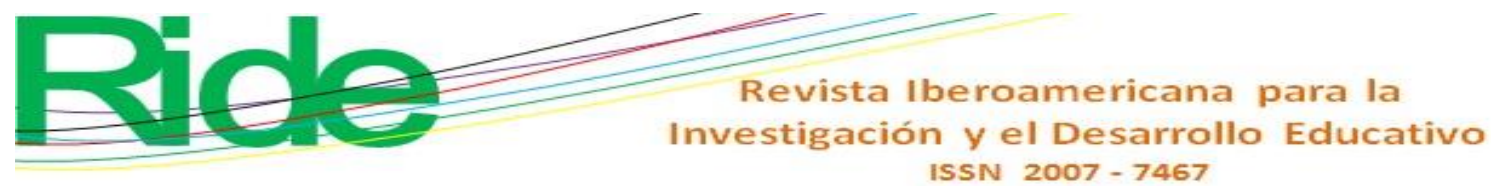

Por el incremento en la calidad educativa, la capacitación y formación de los docentes es una tarea permanente, por lo que - de acuerdo con su formación - se tiene que en el nivel medio superior $64 \%$ de los profesores posee el grado de licenciatura, $2 \%$ cuenta con especialidad y $34 \%$ maestría.

En el nivel superior, $151(22.9 \%)$ tienen el grado de licenciatura, $3(0.5 \%)$ tienen especialidad, 55 (8.4\%) cuentan con especialidad en el área de la salud, 327 (49.7\%) con maestría, y $21(18.5 \%)$ poseen el grado de doctor. Es decir, $77.1 \%$ de la planta docente que imparte en el nivel superior cuenta con estudios de posgrado. Asimismo, del total de PTC, $93 \%$ se encuentran registrados en el Programa para el Desarrollo Profesional Docente [Prodet] de la Secretaría de Educación Pública.

\section{Metodología}

La presente investigación (derivada de un proyecto de tesis doctoral) se sustentó en la metodología cualitativa y se llevó a cabo mediante un estudio de caso que permitió un primer acercamiento al tema de estudio elegido. Para ello se diseñó una encuesta bajo tres dimensiones (social, institucional y didáctica), que fueron construidas con base en 87 ítems y que ofrecían cinco opciones de respuesta; cada uno en una escala Likert que va desde Muy de acuerdo hasta Muy en desacuerdo. Para este trabajo, sin embargo, sólo se ofrecen los resultados de ítems significativos que proporcionan información en torno a la perspectiva de los docentes dirigida hacia la compresión de la diversidad, así como las necesidades de cambios en la política educativa y de transformación en la práctica docente. En concreto, los ítems seleccionados fueron los siguientes: 1, 3 y 12 para la dimensión social; 25, 26, 29, 36, 38, 43, 49, 56 y 57 para la dimensión institucional; y 60 y 62 para la dimensión didáctica.

El instrumento usado arrojó un índice de validez mediante el alfa de Cronbach de 0.807, valor que se consiguió a través del análisis estadístico descriptivo de los datos procesados con el software estadístico Statistical Package for the Social Sciences (SPSS).

En la investigación participaron 11 profesores (tres hombres y nueve mujeres) que contaban con los siguientes estudios universitarios:

- Una profesora con nivel licenciatura titulada.

- Dos pasantes o candidatos doctorales.

- Tres con el nivel de doctor. 


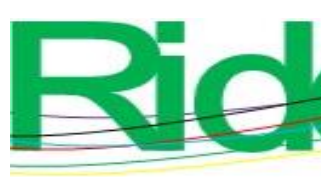

\section{Revista Iberoamericana para la Investigación y el Desarrollo Educativo ISSN 2007 - 7467}

- Seis con el nivel de maestría.

En cuanto a los lugares de trabajo, ocho imparten clases en el nivel superior solamente; uno trabaja en los niveles medio superior y superior, y dos en el nivel medio superior.

Asimismo, es importante mencionar que solo cuatro docentes de los nueve que laboran en el nivel superior participan en Carrera Docente, programa que estimula mediante recursos económicos la labor magisterial que realizan. Este consta de acciones tutoriales, gestión académica, investigación y clases frente a grupo.

\section{Resultados}

A continuación, se ofrecen los resultados conseguidos en cada una de las dimensiones seleccionadas en el presente trabajo. En primer lugar, en las tablas 1, 2 y 3 se muestran los hallazgos recabados con los ítems 1,3 y 12 (dimensión social):

\section{Dimensión social: Comprensión de la diversidad y práctica docente}

Los datos ofrecidos en las tablas 2 y 3 permiten afirmar que los docentes son conscientes de las diferencias que existen entre los estudiantes, así como de la necesidad atender a todos sin importar el origen, las condiciones sociales, los factores étnicos o las deficiencias físicas o mentales.

En la tabla 4, en cambio, se observa que la mayoría de los participantes están de acuerdo o muy de acuerdo con ayudar a las personas con capacidades diferentes cuando no puedan realizar actividades.

Tabla 2. Ítem 1. Todos somos diferentes en capacidades, costumbres e historia de vida

\begin{tabular}{|l|l|r|r|r|r|}
\hline & & Frecuencia & Porcentaje & $\begin{array}{c}\text { Porcentaje } \\
\text { válido }\end{array}$ & $\begin{array}{c}\text { Porcentaje } \\
\text { acumulado }\end{array}$ \\
\hline Válido & DE ACUERDO & 4 & 36.4 & 36.4 & 36.4 \\
\hline & MUY DE ACUERDO & 7 & 63.6 & 63.6 & 100.0 \\
\hline & Total & 11 & 100.0 & 100.0 & \\
\hline
\end{tabular}

Fuente: Elaboración propia 


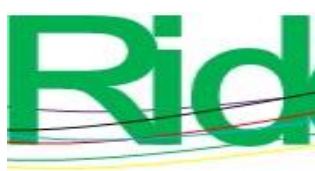

Revista Iberoamericana para la Investigación y el Desarrollo Educativo ISSN 2007 - 7467

Tabla 3. Ítem 3. Existen diferentes formas de ser de acuerdo con las familias que los encausa

\begin{tabular}{|l|l|r|r|r|r|}
\hline & Frecuencia & Porcentaje & $\begin{array}{c}\text { Porcentaje } \\
\text { válido }\end{array}$ & $\begin{array}{c}\text { Porcentaje } \\
\text { acumulado }\end{array}$ \\
\hline Válido & EN DESACUERDO & 18.2 & 18.2 & 18.2 \\
\hline & $\begin{array}{l}\text { NI DE ACUERDO NI EN } \\
\text { DESACUERDO }\end{array}$ & 3 & 27.3 & 27.3 & 45.5 \\
\hline & DE ACUERDO & 5 & 45.5 & 45.5 & 90.9 \\
\hline & MUY DE ACUERDO & 1 & 9.1 & 9.1 & 100.0 \\
\hline & Total & 11 & 100.0 & 100.0 & \\
\hline
\end{tabular}

Fuente: Elaboración propia

Tabla 4. Ítem 12. Si las personas con capacidades diferentes no pueden realizar actividades, debo ayudarlas

\begin{tabular}{|l|l|r|r|r|r|}
\hline & & Frecuencia & Porcentaje & $\begin{array}{c}\text { Porcentaje } \\
\text { válido }\end{array}$ & $\begin{array}{c}\text { Porcentaje } \\
\text { acumulado }\end{array}$ \\
\hline Válido & EN DESACUERDO & 1 & 9.1 & 9.1 & 9.1 \\
\hline & $\begin{array}{l}\text { NI DE ACUERDO NI EN } \\
\text { DESACUERDO }\end{array}$ & 3 & 27.3 & 27.3 & 36.4 \\
\hline & DE ACUERDO & 5 & 45.5 & 45.5 & 81.8 \\
\hline & MUY DE ACUERDO & 2 & 18.2 & 18.2 & 100.0 \\
\hline & Total & 11 & 100.0 & 100.0 & \\
\hline
\end{tabular}

Fuente: Elaboración propia

\section{Dimensión institucional: Cambios en la política educativa, articulación en niveles educativos y retos docentes}

Esta dimensión institucional se enfocó en las implicaciones del sistema educativo en cuanto a las transformaciones sociales que se afrontan y en las necesidades de actualización profesional. En concreto, se puede indicar que $45 \%$ de los entrevistados apoyan los cambios y las reformas educativas que deben realizarse para fomentar la inclusión de personas con NEE. Asimismo, 27 \% considera que los programas educativos están desvinculados (tabla 6) y a $18 \%$ le resulta frustrante la educación especial (tabla 7). 
Tabla 5. Ítem 25. Por la diversidad que existe en nuestro país, esta reforma educativa se debe dar

\begin{tabular}{|l|l|r|r|r|r|}
\hline & Frecuencia & Porcentaje & $\begin{array}{c}\text { Porcentaje } \\
\text { válido }\end{array}$ & $\begin{array}{r}\text { Porcentaje } \\
\text { acumulado }\end{array}$ \\
\hline Válido & $\begin{array}{l}\text { NI DE ACUERDO NI EN } \\
\text { DESACUERDO }\end{array}$ & 6 & 54.5 & 54.5 & 54.5 \\
\hline & DE ACUERDO & 4 & 36.4 & 36.4 & 90.9 \\
\hline & MUY DE ACUERDO & 1 & 9.1 & 9.1 & 100.0 \\
\hline & Total & 11 & 100.0 & 100.0 & \\
\hline
\end{tabular}

Fuente: Elaboración propia

Tabla 6. Ítem 26. Los niveles educativos están desvinculados

\begin{tabular}{|l|l|r|r|r|r|}
\hline & & Frecuencia & Porcentaje & $\begin{array}{c}\text { Porcentaje } \\
\text { válido }\end{array}$ & $\begin{array}{c}\text { Porcentaje } \\
\text { acumulado }\end{array}$ \\
\hline Válido & MUY EN DESACUERDO & 5 & 45.5 & 45.5 & 45.5 \\
\hline & EN DESACUERDO & 3 & 27.3 & 27.3 & 72.7 \\
\hline \multirow{2}{*}{} & DE ACUERDO & 3 & 27.3 & 27.3 & 100.0 \\
\hline & Total & 11 & 100.0 & 100.0 & \\
\hline
\end{tabular}

Fuente: Elaboración propia

Tabla 7. Ítem 29. La educación especial es frustrante e incongruente en el seguimiento

\begin{tabular}{|l|l|r|r|r|r|}
\hline & Frecuencia & Porcentaje & $\begin{array}{c}\text { Porcentaje } \\
\text { válido }\end{array}$ & $\begin{array}{r}\text { Porcentaje } \\
\text { acumulado }\end{array}$ \\
\hline Válido & MUY EN DESACUERDO & 1 & 9.1 & 9.1 & 9.1 \\
\hline & EN DESACUERDO & 1 & 9.1 & 9.1 & 18.2 \\
\hline & $\begin{array}{l}\text { NI DE ACUERDO NI EN } \\
\text { DESACUERDO }\end{array}$ & 7 & 63.6 & 63.6 & 81.8 \\
\hline & DE ACUERDO & 2 & 18.2 & 18.2 & 100.0 \\
\hline & Total & 11 & 100.0 & 100.0 & \\
\hline
\end{tabular}

Fuente: Elaboración propia

En esta misma dimensión institucional, pero refiriéndose a la política educativa relacionada con la experiencia profesional, se les preguntó a los participantes si había resistencia al cambio, ante a lo cual $81 \%$ se mostró entre muy en desacuerdo y en desacuerdo (tabla 8), mientras que $18 \%$ sí cree tener resistencia a ese cambio. Además, $90 \%$ lo ve como un reto personal (tabla 9) y $45 \%$ concluye que no sabe nada de educación especial (tabla 10). 
Tabla 8. Ítem 33. Existe resistencia al cambio, aunque haya actualización o capacitación docente

\begin{tabular}{|c|c|c|c|c|c|}
\hline & & Frecuencia & Porcentaje & $\begin{array}{c}\text { Porcentaje } \\
\text { válido }\end{array}$ & $\begin{array}{l}\text { Porcentaje } \\
\text { acumulado }\end{array}$ \\
\hline Válido & MUY EN DESACUERDO & 2 & 18.2 & 18.2 & 18.2 \\
\hline & EN DESACUERDO & 7 & 63.6 & 63.6 & 81.8 \\
\hline & MUY DE ACUERDO & 2 & 18.2 & 18.2 & 100.0 \\
\hline & Total & 11 & 100.0 & 100.0 & \\
\hline
\end{tabular}

Fuente: Elaboración propia

Tabla 9. Ítem 38. Es un reto profesional como docente

\begin{tabular}{|l|l|r|r|r|r|}
\hline & & Frecuencia & Porcentaje & $\begin{array}{c}\text { Porcentaje } \\
\text { válido }\end{array}$ & $\begin{array}{c}\text { Porcentaje } \\
\text { acumulado }\end{array}$ \\
\hline Válido & $\begin{array}{l}\text { NI DE ACUERDO NI EN } \\
\text { DESACUERDO }\end{array}$ & 1 & 9.1 & 9.1 & 9.1 \\
\hline & DE ACUERDO & 6 & 54.5 & 54.5 & 63.6 \\
\hline & MUY DE ACUERDO & 4 & 36.4 & 36.4 & 100.0 \\
\hline & Total & 11 & 100.0 & 100.0 & \\
\hline
\end{tabular}

Fuente: Elaboración propia

Tabla 10. Ítem 43. Se concluye que como docente no sabemos nada de educación especial

\begin{tabular}{|l|l|r|r|r|r|}
\hline & & Frecuencia & Porcentaje & $\begin{array}{c}\text { Porcentaje } \\
\text { válido }\end{array}$ & $\begin{array}{c}\text { Porcentaje } \\
\text { acumulado }\end{array}$ \\
\hline Válido & MUY EN DESACUERDO & 2 & 18.2 & 18.2 & 18.2 \\
\hline & EN DESACUERDO & 2 & 18.2 & 18.2 & 36.4 \\
\hline & $\begin{array}{l}\text { NI DE ACUERDO NI EN } \\
\text { DESACUERDO }\end{array}$ & 2 & 18.2 & 18.2 & 54.5 \\
\hline & DE ACUERDO & 5 & 45.5 & 45.5 & 100.0 \\
\hline & Total & 11 & 100.0 & 100.0 & \\
\hline
\end{tabular}

Fuente: Elaboración propia

Ahora bien, llama la atención en la tabla 11 que casi $36 \%$ de los entrevistados muestra su descuerdo al momento que se les cuestiona sobre si la UACAM no tiene dominio sobre el enfoque de NEE, pues en la tabla 12 se aprecia que $72.7 \%$ de los docentes se consideran tradicionalistas. Además, $54 \%$ tiene muy arraigados los programas anteriores (tabla 13). 


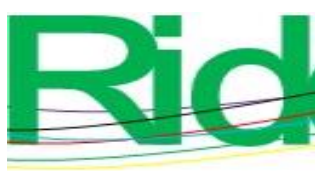

Revista Iberoamericana para la Investigación y el Desarrollo Educativo ISSN 2007 - 7467

Tabla 11. Ítem 49. Es claro que nuestra institución no tiene dominio sobre este enfoque

\begin{tabular}{|l|l|r|r|r|r|}
\hline & & Frecuencia & Porcentaje & $\begin{array}{c}\text { Porcentaje } \\
\text { válido }\end{array}$ & $\begin{array}{c}\text { Porcentaje } \\
\text { acumulado }\end{array}$ \\
\hline Válido & MUY EN DESACUERDO & 2 & 18.2 & 18.2 & 18.2 \\
\hline & EN DESACUERDO & 2 & 18.2 & 18.2 & 36.4 \\
\hline & $\begin{array}{l}\text { NI DE ACUERDO NI EN } \\
\text { DESACUERDO }\end{array}$ & 6 & 54.5 & 54.5 & 90.9 \\
\hline & DE ACUERDO & 1 & 9.1 & 9.1 & 100.0 \\
\hline & Total & 11 & 100.0 & 100.0 & \\
\hline
\end{tabular}

Fuente: Elaboración propia

Tabla 12. Ítem. 56. Me considero tradicionalista

\begin{tabular}{|l|l|r|r|r|r|}
\hline & & Frecuencia & Porcentaje & $\begin{array}{c}\text { Porcentaje } \\
\text { válido }\end{array}$ & $\begin{array}{c}\text { Porcentaje } \\
\text { acumulado }\end{array}$ \\
\hline Válido & EN DESACUERDO & 2 & 18.2 & 18.2 & 18.2 \\
\hline & $\begin{array}{l}\text { NI DE ACUERDO NI EN } \\
\text { DESACUERDO }\end{array}$ & 1 & 9.1 & 9.1 & 27.3 \\
\hline & DE ACUERDO & 7 & 63.6 & 63.6 & 90.9 \\
\hline & MUY DE ACUERDO & 1 & 9.1 & 9.1 & 100.0 \\
\hline & Total & 11 & 100.0 & 100.0 & \\
\hline
\end{tabular}

Fuente: Elaboración propia

Tabla 13. Ítem 57. Tengo muy arraigados los programas educativos anteriores

\begin{tabular}{|l|l|r|r|r|r|}
\hline & Frecuencia & Porcentaje & $\begin{array}{c}\text { Porcentaje } \\
\text { válido }\end{array}$ & $\begin{array}{r}\text { Porcentaje } \\
\text { acumulado }\end{array}$ \\
\hline Válido & MUY EN DESACUERDO & 1 & 9.1 & 9.1 & 9.1 \\
\hline & $\begin{array}{l}\text { NI DE ACUERDO NI EN } \\
\text { DESACUERDO }\end{array}$ & 4 & 36.4 & 36.4 & 45.5 \\
\hline & DE ACUERDO & 6 & 54.5 & 54.5 & 100.0 \\
\hline & Total & 11 & 100.0 & 100.0 & \\
\hline
\end{tabular}

Fuente: Elaboración propia 


\section{Dimensión didáctica: Transformación de la práctica docente en el contexto educativo}

En esta dimensión se consideraron los enfoques hacia las competencias y cómo influyen en la percepción docente, así como de las acciones que hacen o dejan de hacer en el momento en que se encuentran con estudiantes con NEE dentro del aula. Al respecto, se puede decir que el $45.6 \%$ de los profesores se muestran en desacuerdo y muy en desacuerdo cuando se les preguntó si se les hacía difícil entender el concepto de competencias para personas con NEE (tabla 14). Asimismo, $82 \%$ se muestra de acuerdo y muy de acuerdo hacia el cambio de la práctica cuando hay en el aula estudiantes con NEE (tabla 15).

Tabla 14. Ítem 60. Es difícil entender el concepto de competencias para personas con NEE

\begin{tabular}{|c|c|c|c|c|c|}
\hline & & Frecuencia & Porcentaje & $\begin{array}{c}\text { Porcentaje } \\
\text { válido }\end{array}$ & $\begin{array}{l}\text { Porcentaje } \\
\text { acumulado }\end{array}$ \\
\hline \multirow[t]{5}{*}{ Válido } & MUY EN DESACUERDO & 2 & 18.2 & 18.2 & 18.2 \\
\hline & EN DESACUERDO & 3 & 27.3 & 27.3 & 45.5 \\
\hline & $\begin{array}{l}\text { NI DE ACUERDO NI EN } \\
\text { DESACUERDO }\end{array}$ & 2 & 18.2 & 18.2 & 63.6 \\
\hline & DE ACUERDO & 4 & 36.4 & 36.4 & 100.0 \\
\hline & Total & 11 & 100.0 & 100.0 & \\
\hline
\end{tabular}

Fuente: Elaboración propia

Tabla 15. Ítem 62. Se debe cambiar mi práctica docente ante estudiantes con NEE

\begin{tabular}{|l|l|r|r|r|r|}
\hline & Frecuencia & Porcentaje & $\begin{array}{c}\text { Porcentaje } \\
\text { válido }\end{array}$ & $\begin{array}{c}\text { Porcentaje } \\
\text { acumulado }\end{array}$ \\
\hline Válido & $\begin{array}{l}\text { NI DE ACUERDO NI EN } \\
\text { DESACUERDO }\end{array}$ & 2 & 18.2 & 18.2 & 18.2 \\
\hline & DE ACUERDO & 5 & 45.5 & 45.5 & 63.6 \\
\hline & MUY DE ACUERDO & 4 & 36.4 & 36.4 & 100.0 \\
\hline & Total & 11 & 100.0 & 100.0 & \\
\hline
\end{tabular}

Fuente: Elaboración propia 

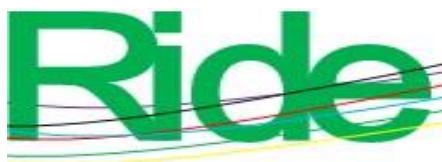

Revista Iberoamericana para la
Investigación y el Desarrollo Educativo

ISSN 2007 - 7467

organismos acreditadores. Tecnología Educativa. Revista CONAIC, 4(12), 35-51. https://tinyurl.com/y3d6fs2y

Universidad Autónoma de Campeche [UACAM] (2007). Lineamientos de transparencia y acceso a la información pública de la Universidad Autónoma de Campeche. Recuperado de https://tinyurl.com/y2nth2la

Universidad Autónoma de Campeche [UACAM] (2015-2019). Plan Institucional de Desarrollo 2015-2019. Recuperado de https://tinyurl.com/ybgg4wz7

Universidad Autónoma de Campeche [UACAM] (2018-2019). 4to. Informe de Actividades 20182019. Recuperado de https://tinyurl.com/yy2qzrvv

Vergara, J. (2002). Marco histórico de la educación especial. Revista Estudios, (2), 129-143. https://tinyurl.com/y86mxrkl 